\title{
MASTER
}

UCRL-\$2634

\section{ELECTRON CYCLOTRON RESONANCE HEATING OF TANDEM MIRRORS}

Miklos Porkolab

December 19,1978

Work performed under the auspices of the US Department of

Energy by the UCLLL under contract number W-7405-ENG.4B

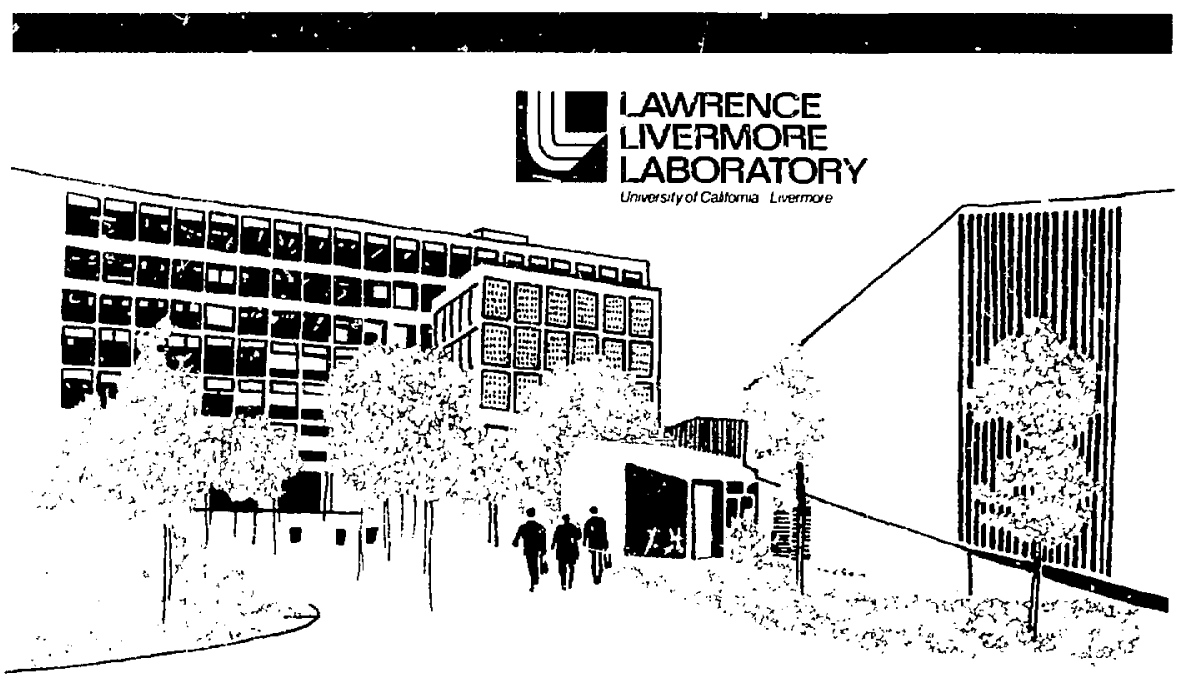


Distribution Category

uc -20

\author{
UCRL-52634 \\ ELECTRON CYCLOTRON RESONANCE HEATIYY. \\ OF TANDEM MIRRORS \\ Miklos Porkolab*
}

MS. date: December 19,1978

* Permanent address: Massachusetts Institute of Technology, Cambridge, MA 02139 


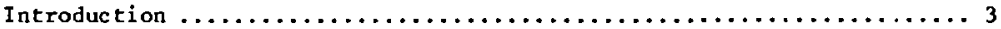

Physics of Wave Absorption and Accessibility .................6

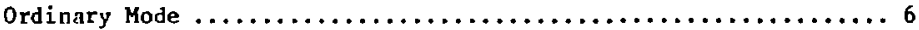

Extraordinary Mode $\ldots \ldots \ldots \ldots \ldots \ldots \ldots \ldots \ldots \ldots \ldots \ldots \ldots \ldots$

Accessibility $\ldots \ldots \ldots \ldots \ldots \ldots \ldots \ldots \ldots \ldots \ldots \ldots \ldots$

Absorption Lengths $\ldots \ldots \ldots \ldots \ldots \ldots \ldots \ldots \ldots \ldots \ldots$

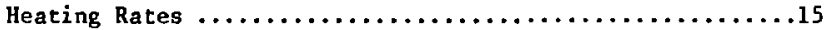

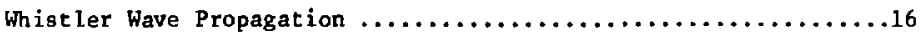

Heating of Tandem Mirrors $\ldots \ldots \ldots \ldots \ldots \ldots \ldots \ldots \ldots \ldots \ldots \ldots \ldots \ldots \ldots \ldots$

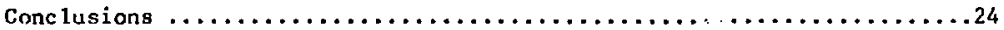

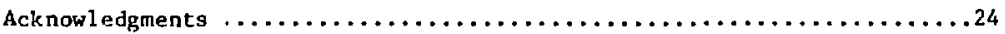

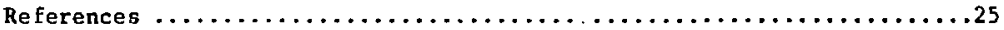




\section{ELECTRON CYCLOTRON RESONANCE HEATING OF TANDEM MIRRORS}

\section{ABSTRACT}

Heating electrons by microwave power near either the electron cyclotron frequency or its harmonic is analyzed in some detail. The purpos? of $\mathrm{Ln}$ is study is to determine whether electrons in current or future mirror devices could be heated by electromagnetic waves in this frequency range. Absorption lengths, accessibility conditions, and appropriate polarization are among the concerns stu ied. It is found that the Tandem Mirror Experiment (TMX) may efficiently be heated by the extraordinary mode at $\omega=\omega_{c e}$ either in the solenoid-to-plug transition region or near the center of the plug at densities $n \approx 10^{13} \mathrm{~cm}^{-3}$.

\section{INTRODUCTION}

Heating of electrons in a single-cell mirror or a tandem mirror reactor (TMR) is of interest for the following reasons:

- By creat:ing energetic electrons in a single-cell mirror for in the end plugs of a tandem mirror), we may be able to alter the potential locally so that low-energy ions can be trapped and their lifetime thereby increased. This would improve stabilization of the drift-cyclotron loss-cone (DCLC) mode and would allow a reduction of cold gas flow. 1

- As shown in Fig. 1, heating the bulk of the electrons in the central ce:! if a TMR would allow a reduction of the beam energies injectad into the plug to about $0.5 \mathrm{MeV}$ (froja above $1 \mathrm{MeV}$ ), would reduce the total cost. and would reduce the magnetic field in the $p^{2} \mathrm{ug}^{2}$ from about $17 \mathrm{~T}$ to $12 \mathrm{~T}$. In addition, the electron energy confinement time in the central solenoid, as well as the overall Q, (defined as the ratio of fusion power output to the neutral-beam power injected to sustain the reaction) should be enhanced.

All of these factors would improve the potential performance of tandem mirror devices as reactors. 


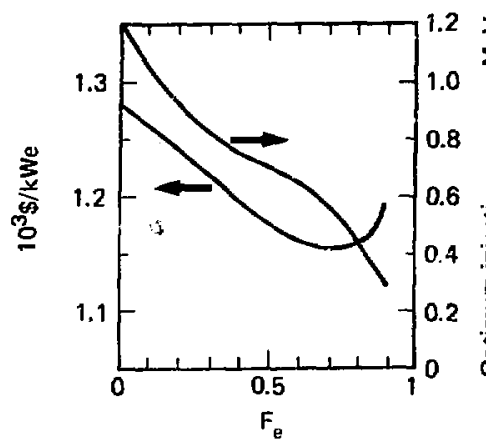

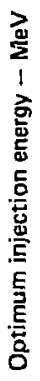

Fig. 1. Cost of a tandem mirror reactor va fraction of heating direct to electrons.

We have examined the following frequency regimes for heating electrons in a tandem mirror device: (a) the electron cyclotron frequency (or its harmonic), (b) the lower-hybrid frequency, and (c) the fast magnetosonic wave or the Alfven wave. For the present and the near future, the best frequency range appears to be that near the electron, cyclotron frequency or its harmonic. From the long-range (reactor) point of view, heating with the compressional Alfuén wave also appears favorable. Lower-hybrid wave heating has the disadvantage of depositing the energy too far out in the tail of the distribution function $\left(v_{p h} / v_{t e} \approx 3\right.$ or $\left.\mathrm{W} / \mathrm{nT}_{\mathrm{e}} \approx 9\right)$ where confinement is no longer provided by the end plugs (unless this tail is thermalized in times short compared with the transit time of the energetic electrons). Other electron heating methods such as electron-beam/plasma interaction or ion-cyclotron heating have not been examined in this study.

Heating bulk electrons is possible using the fast magnetosonic wave $\left(\omega>\omega_{c i}\right)$ or, if a parallel wavelength of the order of $w / k_{\|} \approx v_{t e}$ is imposed by external loops, ${ }^{3}$ the compressional Alfven wave ( $\omega<\omega_{c i}$ ). Although this technique is favorable at high temperatures $\left(T_{e}>1\right.$ keV) and in long devices (where $L \approx 100 \mathrm{~m}$, as in the $T M R$ ), in present-day devices the absorption length is too long to be of interest. 4

For these reasons, perhaps the most promising (although not necessarily the cheapest) technique for heating electrons in both present and future mirror devices (including tandem mirrors) appears to be near the electron cyclotron frequency or ite harmonic. This conciusion is further supported by the consideration of a new generation of high-power microwave devices, 
namely gyrotrons, that will soon be available in the 28 - to 35-GHz Erequency range at power levels of $200 \mathrm{~kW}$ per tube and pulse lengths of $10 \mathrm{~ms}$ to CW. $5-7$

Since cyclotron resonance is at $f(\mathrm{GHz})=28 \vee B_{T}(T)$ (where $v=1$ for

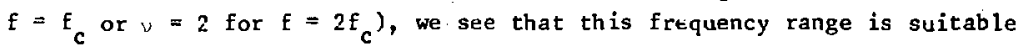
for heating at the fundamental at $B \approx 1 \mathrm{~T}$, and at the harmonic at $\mathrm{B} \approx 0.5 \mathrm{~T}$. Thus, these sources could be used to heat a tandem mirror such as the Tandem Mirror Experiment (TMX) either near the middle of the plug or near the rising part of the magnetic field between the snlenoid and the plug. This question will he examined in some detail in this report.

The next question concerns the future development of gyrotrons. It appears that gyrotrons with frequencies $f \approx 100 \mathrm{GHz}$ and power levels of $P \approx$ $100 \mathrm{~kW}$ will become a reality within the next 3 years. 8 This frequency would be appropriate to heat plasmas either at $B \approx 3.5 \mathrm{~T}$ at $\mathrm{f}=\mathrm{f}_{\mathrm{ce}}$ or at $1.8 \mathrm{~T}$ at $\mathrm{f}=2 \mathrm{f}_{\mathrm{ce}}$. These parameters may be of interest for the central cell of tandem mirror reactors. We note that although the efficiency of these tubes is now 30\%, in principle, $70 \%$ efficiency could be obtained by advanced design Lechniques. However, such a technology remains to be proven, especially if power of the order of 50 to $100 \mathrm{MW}$ is required to heat a reactor.

Recent experimental results on the TM-3 tokamak showed that heating at $f=f_{c e}$ or $f=2 f_{c e}$ could be obtained with absorption efficiencies of the order of $n \approx 0.30$ (where $n=\Delta\left(n T_{e}\right) / P_{\text {source }}$ ). ${ }^{9}$ These results were obtained on TM-3 under conditions that were not necessarily optimum from the point of view nf plasma heating. In principle, with careful design higher heating efficiencies should be obtainable (perhaps of the order of $n \approx 0.70$ ).

We wish to emphasize that in the present work we are investigating heating relatively high-density plasmas $\left(n \approx 10^{13} \mathrm{~cm}^{-3}\right)$; unlike in earlier low-density experiment, $\left(n<10^{12} \mathrm{~cm}^{-3}\right.$ ), we must carefully evaluate questions such as accessibility and wave absorption. In particular, although penetration is relatively easy at low densities and long wavelengths, and absorption may proceed via stochastic processes in a multimodecavity configuration ${ }^{10-13}$ (which may lead to a loss-cone distribution for electrons ${ }^{14,15}$ ), in the present regime the densities are high, the wavelengths are short, and therefore accessibility must be satisfied. The absorption mechanism relevant to this regime is similar to that used in recent tokamak work, namely, cyclotron absorption in one pass in an inhomog- 
eneous magnetic field. ${ }^{15-19}$ This type of heating may lead to a Maxwellian electron distribution (due to collisional isotropization) as observed in recent tokamak experiments. 9

THE PHYSICS OF JAVE ABSORPTION AND ACCESSIBILITY

We shall now present detailed calculations on wave absorption and accessibility. For this discussion, we assume that the polarization and/or the angle of incidence can be varied by using appropriate microwave horns $\left(\mathrm{N}_{2}, 1\right)$ and/or waveguide arrays $\left(\mathrm{N}_{2}>1\right)$.

ORD INARY MODE

In the ordinary mode ( 0 -mode), the wave is incident along the $x-$ direction, normal to the external magnetic field $B_{0} \hat{z}$, ( $E \| B_{0}$ ), and accessibility exists only if $f_{0}{ }^{-} f_{p e}$, where $f_{p e}$ is the local electron plasma Erequency. 20 Since for absorption we need $f_{0}=f_{c e}$, the condition for ahsorption and recessibility is

$$
\mathrm{f}_{0}=\mathrm{f}_{\mathrm{ce}}-\mathrm{E}_{\mathrm{pe}}
$$

This translates into the following density limit for penetration at a given magnetic field:

$$
\mathrm{n}_{13}<0.97 \nu^{2} \mathrm{~B}_{\mathrm{T}}^{2}
$$

where $\nu=1$ or 2 for $f=E_{c}$ or $f=2 f_{c e}$, respectively, $n_{13}$ is the plasma density in units of $10^{13} \mathrm{~cm}^{-3}$, and $B_{T}$ is the magnetic field in units of tesla. Since absorption is negligibly small at the second harmonic (unless $\mathrm{T}_{\mathrm{e}} \cdots 10 \mathrm{keV}$ ), for all practical purposes we take $\nu=1\left(\mathrm{f}=\mathrm{f}_{c e}\right)$. Thus, we see that for a magnetic field of $1 \mathrm{~T}$, the density at the resonance layer cannot exceed the value of $\mathrm{n} \approx 10^{13} \mathrm{~cm}^{-3}$, or the wave is reflected.

The absorption coefficient can be de Iined as $^{19}$

$$
A=1-T, \quad T=\exp \left(-\Gamma_{0}\right)
$$


where $T$ is the wave energy transmission coefficient, and $l_{0}$ characterizes the absorption as the wave propagates through the cyclotron resorance layer in the inhomogeneous magnetic field. If we designate the scale length of the magnetic field gradient at the resonance layer by $L_{B}=\left|B(d B / d X)^{-1}\right|$, we have 19

$$
\Gamma_{0}=2 \pi L_{B} \frac{w}{c} \frac{T_{e}}{m_{e} c^{2}} \quad Q_{0},
$$

where

$$
Q_{0}=\frac{1}{4} \frac{\alpha^{2}\left(1-\alpha^{2}\right)^{1 / 2}}{1+N_{z}^{2}\left(1-\alpha^{2}\right)} .
$$

Here, $\alpha^{2}=\omega_{\mathrm{pe}}^{2} / \omega_{0}^{2}, \mathrm{~N}_{\mathrm{z}}=\mathrm{ck}_{\mathrm{z}} / \omega_{0}$ is the component of the index of refraction along the $\ddot{z}$ direction (i.e., along the external magnetic field), and $\mathrm{T}_{\mathrm{e}}$ is the electron temperature. Numerically, we find

$$
\mathrm{r}_{0}=2.56 \times 10^{-6} \mathrm{~L}_{\mathrm{B}}(\mathrm{cm}) \mathrm{F}(\mathrm{GHz}) \mathrm{T}_{\mathrm{e}}(\mathrm{eV}) \mathrm{Q}_{0} \text {, }
$$

where $L_{B}$ is measured in units of $\mathrm{cm}, f$ is measured in $\mathrm{GHz}$, and $\mathrm{T}_{e}$ is measured in eV. In Fig. 2, we plot numerical values of $Q_{0}$ as a function of $\alpha=\omega_{p e}{ }^{/ \omega_{0}}$ for the 0 -mode for two values of $\mathrm{N}_{z}$, namely, $\mathrm{N}_{\mathbf{z}}=0$ and $\mathrm{N}_{\mathbf{z}}=0.5$. We see that maximum absorption is attained at $\alpha \approx 0.85$, and that above $\alpha \approx 0.95$ and below $\alpha \approx 0.4$ the absorption decreases rapidly. We note that the absorption is only weakly dependent upon $\mathrm{N}_{z}$ (note that $\mathrm{N}_{z}=0$ corresponds to normal incidence). In Fig. 3, we plot absorption coefficients for two types of machines, that corresponding to the central cell of the TMR and that corresponding to the plug of TMX. These curves are obtained for a single pass and one transition through the resonance zone. Actually, in a given machine if $f>f_{p e}(0)$, the wave would pass through the two resonance zones in one pass and the total absorption coefficient would be

$$
A_{\text {total }}=1-T^{2}=1-(1-A)^{2}=A(2-A),
$$

where $A$ is that given in Fig. 3 (i.e., the absorption of a wave passing through one resonance layer). Similarly, if $f=f_{c e}$ is attained near the 
ceriter, $L_{B}$ is considerably larger than $54 \mathrm{~cm}$, and again more efficient absorption is obtained.

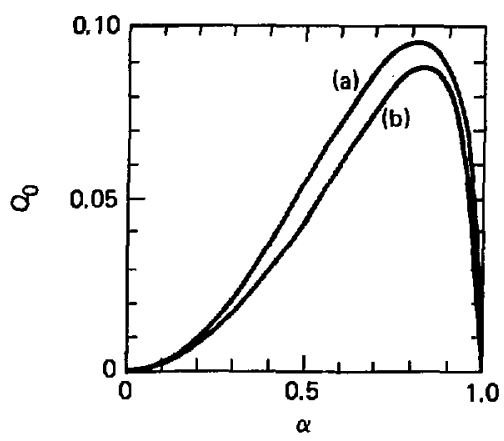

Fig. 2. $Q_{0}$ vs $\alpha$, for 0 -mode, $\omega=$ $\omega_{c e}:$ (a) $N_{z}=0$, and (b) $N_{z}=0.5$.

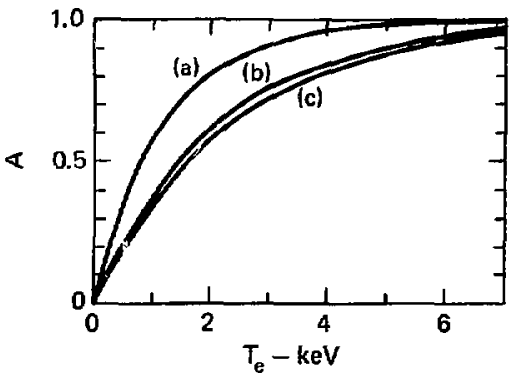

Fig. 3. Absorption coefficient A vs electron temperature $\mathrm{T}_{\mathrm{e}}$ fror 0-mode, $\omega=\omega_{\mathrm{ce}}$, and $\alpha=0.80$ :

(a) TMR curve, $f=70 \mathrm{GHz}, \mathrm{N}_{z}=0$, $\mathrm{L}=50 \mathrm{~cm}, \mathrm{~B}=2.5 \mathrm{~T}, \mathrm{n}=6 \times$ $10^{13} \mathrm{~cm}^{-3}$;

(b) $T M x$ curve, $f=35 \mathrm{GHz}, \mathrm{L}=54 \mathrm{~cm}$, $\mathrm{B}=1.25 \mathrm{~T}, \mathrm{n}=1.5 \times 10^{13}$ $\mathrm{cm}^{-3}, \mathrm{~N}_{\mathrm{z}}=0$; and

(c) same as (b), but $\mathrm{N}_{2}=0.5$.

EXTRAORDINARY MODE

\section{Accessibility}

The extraordinary mode (EO-mode) may be efficiently absorbed at either $f=f_{c e}$ or $f=2 f_{c e}$. Accessibility in a uniform magnetic field and radially increasing density are available only at $f=2 f_{c e}(v=2)$, and the plasma density must not be so high that the cut-off layer 


$$
\omega_{ \pm}= \pm \frac{\omega_{c e}}{2}+\frac{1}{2}\left(\begin{array}{c}
2 \\
\omega_{c e}+4 \omega_{p e}^{2}
\end{array}\right)^{1 / 2}
$$

exceeds the incident frequency, i.e., $\omega_{0}=2 \omega_{c e}>_{+^{*}}$ This means that for accessibility

$$
{ }^{2}{ }_{\mathrm{pe}}^{2} / \omega_{\mathrm{ce}}^{2}<2 \text {, or } \mathrm{n}_{13}<1.95 \mathrm{~B}_{\mathrm{T}}^{2} \text {. }
$$

$\left(\begin{array}{c}2 \\ 2 \\ w_{p e}\end{array}+\omega_{c e}^{2}\right.$
)$^{1 / 2}$ is in the range $\omega_{c e}<\omega_{u h}<\omega_{+} \cdot$

If the magnetic field is decreasing rasially toward the center (as when $B>0$ or when a magnetic we 11 exists), accessibility also exists as long as $\omega_{-}<\omega_{c e}=\omega_{0}$. This also gives $\omega_{p e}^{2} / \omega_{c e}^{2} \overline{<} 2$ for accessibility, as in Eq. (6). This situation is shown schematically in Fig. 4, where we see that although in low- $\beta$ tokamaks the power should be injected from the high magnetic field side (i.e., from inside the torus), in a mirror device in regions where a magnetic well exists propagation from the outside is ensured. However, we also note that in symmetric magnetic wells the upper-hybrid layer is not accessible (unlike in tokamaks, where $\omega_{0}=\omega_{\text {uh }}$ is another potential abscrption layer). We may also note that for a fixed frequency the highest density 1 imit for the EO-mode is

$$
\mathrm{n}_{\max }\left(\mathrm{cm}^{-3}\right)=1.95 \times 10^{13} \frac{\mathrm{f}(\mathrm{GHz})}{28 v}
$$

Thus, the highest accessible density for the E0-mode is at $v=1$ (i.e., $f=$ $\mathrm{f}_{\mathrm{ce}}$ ). However, we shall see that; at the limiting density, the absorption drops sharply so that one may have to contend with densities somewhat below that predicted by Eq. (7).

\section{Absorption Lengths}

We shall consider absorption lengths at the fundamental electron cyclotron frequency and at its hammonic. 

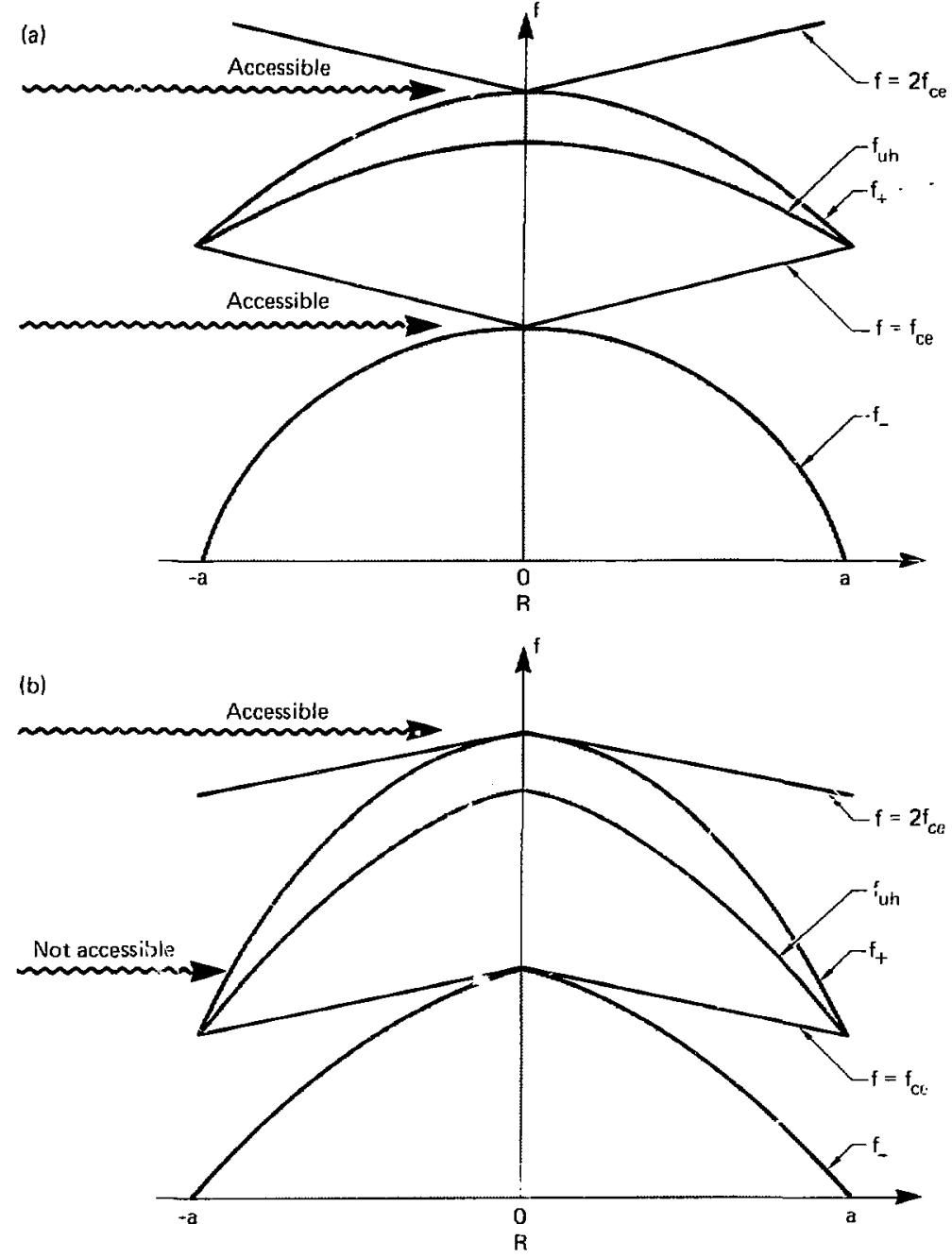

Fig. 4. Sretched accessibility curves for (a) a magnetic field decreasing radially inward and (b) a magnetic field increasing radially inward, for EO-mode, $\omega_{\mathrm{pe}}^{2} / \omega_{c e}^{2}=2, \omega_{+}=2 \omega_{c e}$, and $\omega_{-}=\omega_{c e}$. Shaded areas are regions of evanescence. 
Absorption at the Fundamental Electron Cyclotron Frequency. The absorption at $f=f_{c e}$ is tather ineffective for norinal incidence, and we have for the transmission coefficient in Eq. (2),

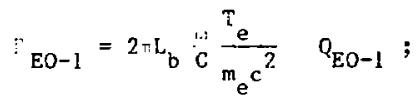

where for $\mathrm{N}_{2}=0$ we have ${ }^{19}$

$$
Q_{E O-1}=\frac{1}{8} \frac{T_{e}}{m_{e} c^{2}} x^{2}\left(2-\alpha^{2}\right)^{3 / 2},
$$

and for $\mathrm{N}_{\mathrm{z}}+0, \mathrm{~N}_{\mathrm{z}}\left(\mathrm{T}_{\mathrm{e}} / \mathrm{m}_{\mathrm{e}} \mathrm{c}^{2}\right)^{1 / 2} \cdots \mathrm{l}$ ( we have 16,18

$$
Q_{E O-1}=\frac{N_{x}^{2}\left(1+a^{2}\right)^{2}\left(2-a^{2}\right)^{3 / 2}}{4: t^{2}} .
$$

For mori general values of the density (i.e., for $n \approx 1$ ) and $\mathrm{w}_{\mathrm{z}}$, the absorption is given by the complicated expressions

$$
q_{E O-1}=\frac{N_{z}^{2}}{i^{2} N_{x}} \frac{\left\{\left(1-\left(\alpha^{2} / 2\right)-N_{z}^{2}\right)\right.}{\left(\left(1-\alpha_{x}^{2}-N_{x}^{2}\right)^{2}+\left(1-\alpha^{2}\right)-N_{x}^{2}\right\}^{2} N_{z}^{2}}
$$

where $\mathrm{N}_{z}$ is fixed by the angle of incidence, and $\mathrm{N}_{x}$ is the cold perpendicular index of refraction; namely,

$$
\mathrm{N}_{\mathrm{x}}^{2}=\frac{\left(1-\alpha^{2}\right)^{2}-r^{2}}{\left(1-a^{2}-y^{2}\right)}
$$

where $a^{2}=\omega_{p e}^{2} / \omega_{0}^{2}$ and $i^{2}=\omega_{c e}^{2} / \omega_{0}^{2}$. From Eq. (g) we note that the absurption of the ro-morie for normal incidence [i.e., Eq. (9)] at $f=f_{\text {ce }}$ is regligibly small for temperatures $T_{e} \approx 10 \mathrm{keV}$ (similar to the 0-mode absorption at $f=2 \mathrm{f}_{c e}$, and we shall ignore this case here. Furthermore, in an actual experiment we may expect $\mathrm{N}_{2}>0$.l due to the finite width of the microwave horn; hence, Eqs. (10) or (ll) are the ones relevant tu experirienta 1 situations. 
In Pig. 5, we plot Eq. (10) for three values of ${ }_{z}$ ar a function of We see that for efficient abeorption at high density (i.e., for . . 1), subatantial values o" $\mathrm{N}_{2}$ are necessary to obtain efficient absorption. Thug, to increage the abeorption efficiency it may be advantagoous to tilt the microwave horn riative to the magnetic field. The Fig. 5 curve also shows the danger of surface absorption in tokamats where cyclotron resonance. exists everywhere across the plasmn column at a fixed value of the major racilug. We also wish to point out from Ref. 18, Fig. 6, that Eq. (11) predicts rapid logs of absorption for ${ }^{2}>1.2$. Thus, for efficient absorption we must assume $i^{2} \sim 1$ (even though penetration exists for ${ }^{2} ; 2$ ).

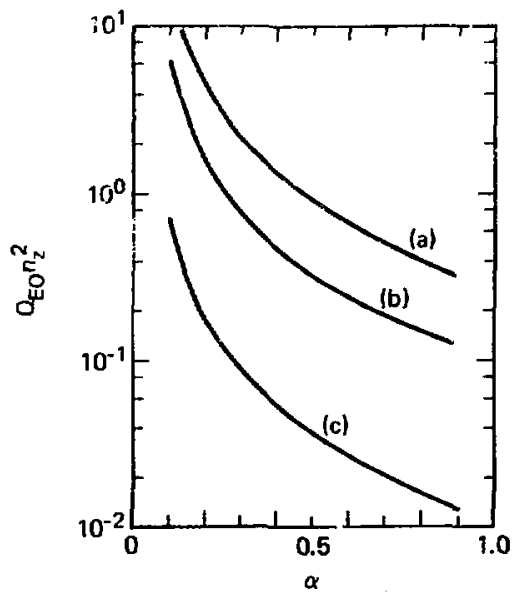

Fig. 5. $Q_{E O} n_{z}^{2}$ vs a for EO-mode, $w=$ $w_{c e}:$
(a) $N_{2}=0.5, \quad \theta \approx 60^{\circ}$;
(b) $\mathrm{N}_{2}=0.3, \quad \theta \approx 72^{\circ}$; and
(c) $\mathrm{N}_{2}=0.1, \quad \theta \approx 84^{\circ}$.

In Figs. 6 and 7 we show absorption curves for $\alpha=0.4$ and 0.8 for three different values of $\mathrm{N}_{2}$ (i.e., for $\mathrm{N}_{2}=0.1,0.3$, and 0.5 ) as a function of electron temperature. These curves can be used in conjunction with present-day gyrotrons, namely, either at $\mathrm{E}=28 \mathrm{GHz}, \mathrm{B}=1.0 \mathrm{~T}, \mathrm{~L}_{\mathrm{B}}=$ $62.5 \mathrm{~cm}$, or at $\mathrm{E}=35 \mathrm{GHz}, \mathrm{B}=1.25 \mathrm{~T}, \mathrm{~L}_{B}=50 \mathrm{~cm}$. The corresponding densities of interest are $n=6.2 \times 10^{12} \mathrm{~cm}^{-3}(\alpha=0.8, f=28 \mathrm{GHz})$ or $n=1.0$ $\times 10^{13} \mathrm{~cm}^{-3}(\alpha=0.8, f=35 \mathrm{GHz})$. For $\alpha=0.4$, the relevant densities are correspondingly lower. Considering $\mathrm{N}_{\mathrm{z}} \approx 0.5, \alpha \approx 1$, we obtain similar curves but approximately $40 \%$ higher densities than obtained for the $a=0.8$ 


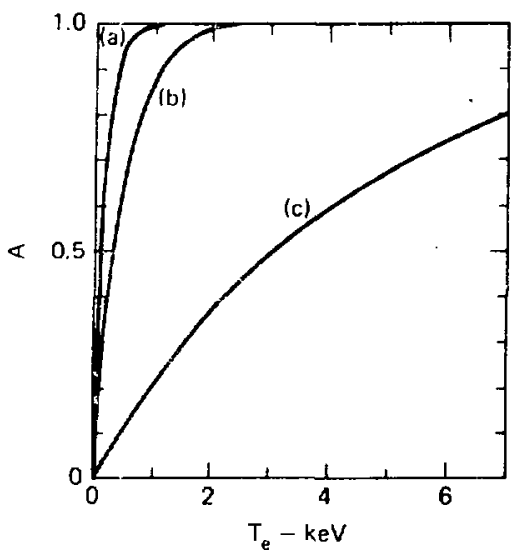

Fig. 6. Absorption coefficient $A$ vs $T_{e}$ for EO-mode, $w=\omega_{c e}, \alpha=0.4, \mathrm{~L}_{B}=$ $50 \mathrm{cr}, \mathrm{f}=35 \mathrm{cHz}$ :
(a) $\mathrm{N}_{2}=0.5\left(0-60^{\circ}\right)$;
(b) $\mathrm{N}_{z}=0.3\left(0=72^{\circ}\right)$; and
(c) $N_{2}=0.1\left(\theta-84^{\circ}\right)$.

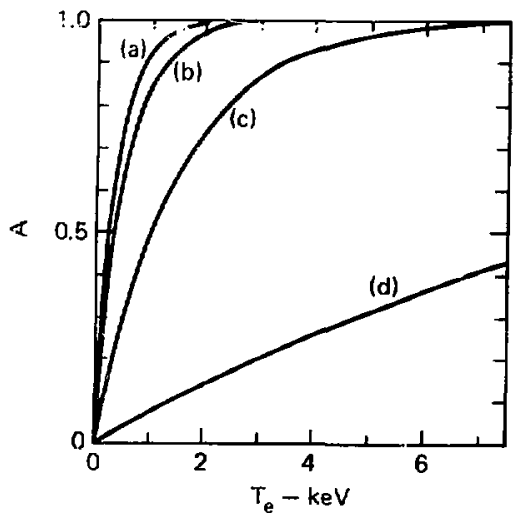

Fig. 7. Absorption coefficient A vs $\mathrm{T}_{\mathrm{e}}$ for EO-mode, $\omega=\omega_{c e}, a=0 . B$ :

(a) $\mathrm{TMX}, \mathrm{f}=35 \mathrm{GHz}, \mathrm{L}_{B}=200 \mathrm{~cm}, \mathrm{~N}_{z}=$ 0.3 ;

(b) $\mathrm{N}_{z}=0.5\left(\theta=60^{\circ}\right)$;

(c) $N_{z}=0.3\left(\theta=72^{\circ}\right)$; and

(d) $\mathrm{N}_{\mathrm{z}}=0.1\left(\theta=34^{\circ}\right)$.

In (b) through (d), $\mathbf{L}_{B}=50 \mathrm{~cm}$, $\mathrm{f}=35 \mathrm{GHz}$.

curves shown here. If we assume that the waves trans te two resonance layers in one pass (as in a magnetic well of a mirror device), correspondingly higher absorption is obtained: namely; $A_{\text {eff }}=A(2-A)$ [see Eq. (4)] . 
Absorption at the Harmonic of the Electron Cyclotron Freguency. At the harmonic of the cyclotron frequency, $f=2 f_{\text {ce }}$, the absorption coefficient is given by 19

$$
\check{E}_{E O-2}=2 \pi I_{B} \frac{T_{e}}{c} \frac{T_{e}}{m_{e} c^{2}} \quad Q_{E O-2} .
$$

For $\mathrm{N}_{\mathrm{z}} \approx 0$ in Eq. (13a), we have

$$
Q_{E O-2}=x^{2}\left[\frac{3-2 x^{2}}{3-4 x^{2}}\right]^{2}\left[\frac{4\left(1-a^{2}\right)^{2}-1}{3-4 x^{2}}\right]^{1 / 2},
$$

where $x^{2}={ }^{2} \mathrm{pe}^{2} / \mathrm{w}^{2}$.

In Fig. 8, we give numerical plots of $\mathrm{Q}_{\mathrm{EO}-2}$ as a function of $x$. We see that the peak absorption occurs at densities such that $\alpha \approx 0.65$ and that the absorption decreases rather rapidly away from this peak yalue. In Fig. 9 , we show absorption curving for some typical $\alpha$ and $L_{B}$ values as a function of electron temperature. These curves are mostly relevant to present-day devices, and are such as may be expected in Thix. We see that the absorption efficiencies increase rapidly with $\mathrm{L}_{B}$ above $\mathrm{T}_{\mathrm{e}} \sim 400 \mathrm{eV}$. We should again emphasize that when the wave passes through two resonance zones the cotal abso ption increases as predicted by Eq. (4). For example, if we take curve (c) in Fig. 9 (i.e., $\mathrm{L}_{\mathrm{B}}=50 \mathrm{~cm}, \alpha=0.60$ ), at $\mathrm{T}_{\mathrm{e}}=400 \mathrm{eVA} \approx 0.60$, and the total efficiency of absorption per pass is $A_{\text {total }}=A(2-A)=0.84$. At $T_{e}=500$ $\mathrm{eV}$, however, $\mathrm{A}=0.70$, so that $A_{\text {total }} \approx 0.91$.

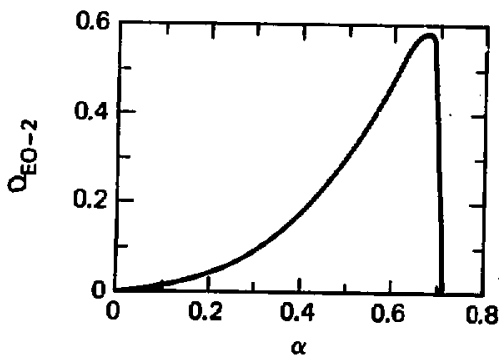

Fig. 8. $Q_{E O-2}$ vB $\propto$ for EO-mode, $\omega=$ ${ }^{2}{ }_{\text {ce }}$. 


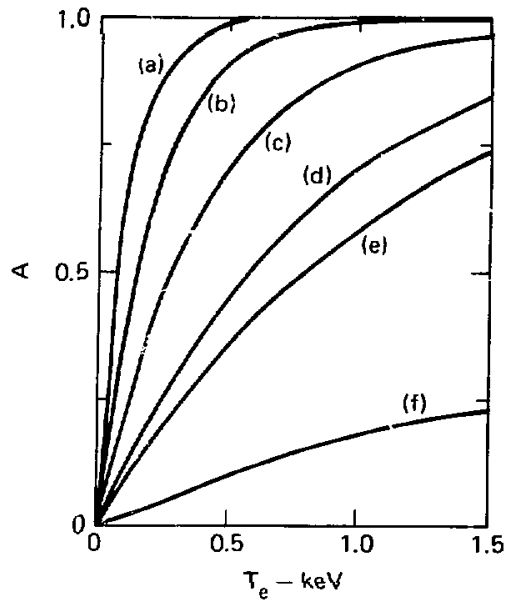

Fig. 9. Absorption coefficient A vs $T_{e}$ ' for E0-mode, $\omega=2 \omega \mathrm{ce}$ :

(a) Th. parameters, $a=0.60, \mathrm{I}_{\mathrm{B}}=50$ $\mathrm{cm}, \mathrm{B}=2.5 \mathrm{~T}, \mathrm{f}=140 \mathrm{GHz}$;

(b) is $=0.60, \mathrm{l}_{\hat{\mathrm{B}}}=100 \mathrm{~cm}$;

(c) ${ }_{13}=0.611, \mathrm{I}_{\mathrm{B}}=50 \mathrm{~cm}$;

(d) ${ }^{\prime} z=0.60, \mathrm{~L}_{B}=25 \mathrm{~cm}$;

(c) ${ }_{\mathrm{I}}=0.40, \mathrm{t}_{\mathrm{B}}=50 \mathrm{~cm}$;

(C) is $=0.20, \mathrm{t}_{\mathrm{B}}=50 \mathrm{~cm}$.

In (b) throuph (f), $r=38$ (H\%.

\section{Heating Rates}

The heating rates have been given by Elridge et al., ${ }^{18}$ and the results may be summarized as follows:

- Perpendicular heating:

$$
\frac{\mathrm{d}^{2} W_{1}}{\mathrm{dtdV}}=\left.\left.\sum_{\operatorname{modes}} \frac{{ }^{2} \mathrm{pe}}{8} \frac{{ }_{c e}}{\omega^{2}} \frac{\mathrm{L}_{B}}{\Delta} \frac{\exp \left(-\mathrm{x}^{2} / 2 \Delta^{2}\right)}{(2 \pi)^{1 / 2}}\right|^{-}\right|^{2} .
$$

- Parallel heating:

$$
\frac{d^{2} W_{\|}}{d t d V}=\sum_{\text {modes }} \frac{\omega_{p e}^{2}}{8 \omega} \frac{x}{\Delta} \frac{\exp \left(-x^{2} / 2 \Delta^{2}\right)}{(2 \pi)^{1 / 2}}\left|E^{-}\right|^{2},
$$

where $E^{-}=E_{x}-i E_{y}$, and

$$
\Delta=N_{z} L_{B}\left(\frac{T_{e}}{m_{e} c^{2}}\right)^{1 / 2}
$$


Here Eq. (15) gives the initial localization of heat deposition. For example, at $T_{e}=1 \mathrm{keV}, L_{B}=100 \mathrm{~cm}_{2} N_{z}=0.5$, and $L=2.2 \mathrm{~cm}$; whereas for $T_{e}$ $=500 \mathrm{eV}, \mathrm{L}_{B}=100 \mathrm{~cm}, \mathrm{~N}_{z}=0.2$, and $\Delta=0.63 \mathrm{~cm}$. Thus, we see that initially the heat deposition is strongly localized. On the other hand, we expect that this heat will spread rapidly due to heat conductivity. We may also conclude that at $f=f_{c e}$, these waves heat bulk electrons, whereas at $\omega=2 \omega$ ce considerable tail heating may also occur.18

A use ful way of looking at heating is to consider the reiationship

$$
P_{\text {abs }}(\text { electron })=\frac{3}{2} \frac{\Delta n T_{e} V}{\tau_{F}}
$$

which tells us the required heating power to achieve a given temperature $\mathrm{T}_{\mathrm{e}}$. In Eq. (16), $\mathrm{T}$ is the energy confinement $t i m e, v$ is the volume, and $\Delta T_{e}$ is the change in electron energy. In the $\mathrm{TXX}$ central cel1, for example if $\mathrm{V} \approx 1.5 \times 10^{3}$ itre, ${ }_{\mathrm{E}} \approx 3 \mathrm{~ms}$, and $\mathrm{n} \approx 1 \times 10^{13} \mathrm{~cm}^{-3}$ with $\mathrm{P}_{\mathrm{abs}}=100 \mathrm{~kW}$ of absorbed power, $\Delta \mathrm{T}_{e} \approx 100 \mathrm{eV}$; and with $0.5 \mathrm{MW}$ of absorbed power, $\Delta \mathrm{T}_{e} \approx 500 \mathrm{eV}$. On the other hand, in the TMR central cell, nt $\approx 8 \times 10^{14} \mathrm{~s} \cdot \mathrm{cm}^{-3}, \mathrm{n}=10^{14} \mathrm{~cm}^{-3}$, and $\mathrm{T}_{\mathrm{e}} \approx 30 \mathrm{keV}$; therefore, $\mathrm{P}_{\text {abs }} \approx 40 \mathrm{MW}$. Assuming $60 \%$ overall efficiency, we see that roughly $70 \mathrm{MW}$ of souree power will be necessary to achieve thes\% electron temperatures (where we considered transmission losses and absorption efficiences).

\section{WHI STLER WAVE PROPAGATION}

Another wave that may be useful for heating mirror devices is the righthand circularly polarized wave, or whistler wave. 20 This wave propagares almost paralle?. to the external magnetic field. The cold-plasua dispersion relationship for parallel prop:gation is given by 20

$$
\mathrm{N}_{\|}^{2}=1-\frac{\omega_{\mathrm{pe}}^{2}}{\omega\left(\omega-\omega \mathrm{ce}^{\top}\right.} \text {. }
$$

In a hot plasma near resonance, $\left|\omega-\omega_{c e}\right|<k_{\|} v_{t e}$, and the dispersion relationship is 10

$$
N_{\|}^{2}=1-\frac{2 \omega_{p e}^{2}}{\omega_{c e}} \frac{\left(\omega-u_{c e}\right)}{k_{\|}^{2} v_{t e}^{2}}+\frac{i \omega_{p e}^{2} \sqrt{\pi}}{\omega_{c e} k_{l} v_{t e}} \exp -\frac{\left(\omega-\omega_{c e}\right)^{2}}{k_{\|}^{2} v_{t e}^{2}},
$$


where $v_{\text {te }}=\left(2 \mathrm{~T}_{\mathrm{e}} / \mathrm{m}_{\mathrm{e}}\right)^{1 / 2}$, and $\mathrm{N}_{\mathrm{f}}^{2}=\mathrm{c}^{2} \mathrm{k}_{\|}^{2 / u^{2}}$.

If we assume that the wave is launched from the high magnetic field side from the mirror plug end such that $w \ll w_{c e}$, as the region $\omega \approx \omega_{c e}$ is approached strong cyclotron absorption sets in. Solving Eq. (18), we obtain

$$
N_{i} \approx\left[\frac{1}{2}+\frac{1}{2}\left(1-\frac{4 c^{2}}{v_{c e}^{2}} \frac{\omega_{p}^{2}}{\omega^{2}} \frac{\left(\omega-\omega_{c e}\right)}{\omega_{c e}}\right)^{1 / 2}\right]^{1 / 2} ;
$$

and at $\omega \approx \omega_{c e}$,

$$
\operatorname{Im} k_{\|} \approx \frac{1}{2}\left(\frac{\pi}{2}\right)^{1 / 2} \frac{\omega_{\mathrm{pe}}^{2}}{v_{\mathrm{te}^{\tan N_{\|}^{2}}}^{2}} .
$$

Thus, we see that at $\omega \approx \omega_{c e}, N_{\|} \approx 1$; and for $\omega \approx \omega_{p e}$, In $k^{-1} \approx 3{ }_{D e}$, which corresponds to very strong absorption. More likely, the wave will be damped out at positions $\omega / \omega_{c e} \approx 0.95$.

The advantage of heating with a whistler wave is the lack of a density limit. A disadvantage may be the difficulty of launching the wave (the microwave horn must be in the high magnetic field, possibly in contact with hot plasme). A second potential problem with wistler waves at high powers is their sensitivity to a number of nonlinear effects, such as parametric decay, ${ }^{22}$ trapping, and self-focusing ${ }^{23}$ while they propagate through the mirror region. We note that some of these effects could lead to the undesirble production of a parallel, superthermal tail of the electron distribution function. 22 Only a thorough experimental test could tell if these nonlinear effects would be strong enough in a given experiment to cause pump-depletion before the wave arrived at the linear absorption region.

\section{HEATING OF TANDEM MIRRORS}

In Fig. 10, we consider the antenna geometries by which single-cell and tandem mirrors may be heated. As discussed before, accessibility requires (a) that $\omega_{p e}{ }^{2} / \omega_{0}{ }^{2} \approx 1$ for the EO-mode (our prime candidate in the present dis- 


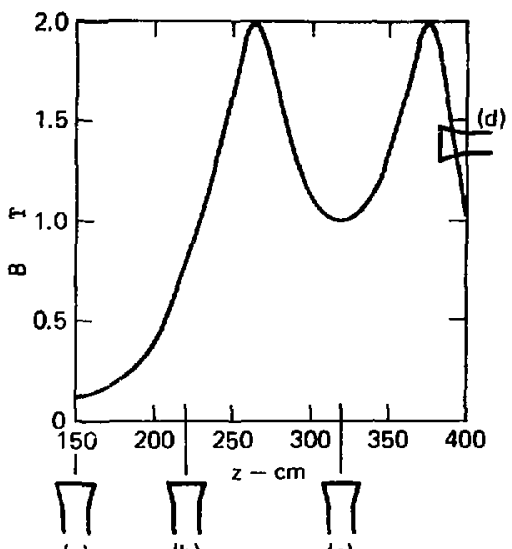

(a)

(b)

(c)
Fig. 10. Axidl plot $c f$ the magnetic field and possible transuitting microwave horn locations for $\beta=0$ :

(a) through (c) for EO-mode, (d) for whist ler mode.

cussion) and (b) that at $\omega_{0}=w_{c e}$ the magnetic field decrease radially inward. In the high- $\beta$ case, these conditions are achieved at any location when a magnetic well exists near the center of the plasma column. In the low- $\beta$ case, accessibility exisis near the positions (a), (b), and (c) in Fig. 10 . This may be seen from piots of the magnetic field lines shown in Fig. 11 , which are representative of the Lawrence Livermore Laboratory TMX device. In Fig. 12, we have plotted the radial variatisn of the vacuum magnetic field intensities in TMX at three different axial locations, namely at $z=$ $150 \mathrm{~cm}, z=220 \mathrm{~cm}$, and $\mathrm{z}=320 \mathrm{~cm}$, corresponding to positions (a), (b), and (c), respectively, in Fig. 11. Figure 13 provides expanded plots for $z=$ $1 j 0 \mathrm{~cm}$ and $z=320 \mathrm{~cm}$. Since these are the vasuum fields, they give typical gradient scale lengths on $1 y$ for low- $\beta$ plasmas. Nevertheless, we can use these as guide lines for our estimates, since for high- $\beta$ plasmas the accessibility for the EO-mode is even better (although the absorption would be more localized to the center where the gradient scale lengths are large).

Using the Fig. 13 curves, in Fig. 14 we plot the radial gradient scale lengths of the magnetic field $L_{B}$. We see thut in both cases $L_{B} \sim 100 \mathrm{~cm}$ for $R<12 \mathrm{~cm}$, and at $\mathrm{R} \approx 5 \mathrm{~cm} \mathrm{~L}_{B} \approx 200 \mathrm{~cm}$ for $z=320 \mathrm{~cm}$ (the center of the mirror plugs) and $L_{B} \approx 10^{3} \mathrm{~cm}$ at $Z=150 \mathrm{~cm}$ (the center solenoid). Foth values of $\mathrm{L}_{B}$ predict strong absorption. 

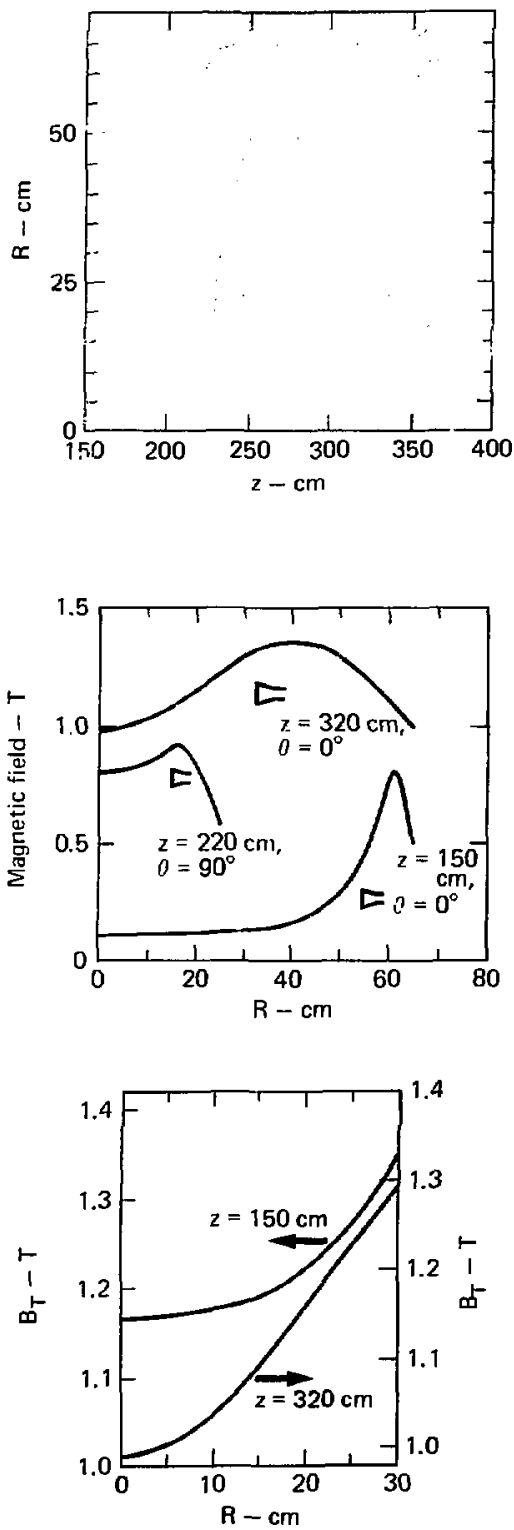

Fig. 11. Contours of the vacuum magnetic field lines in TMX for $\theta=0$. The associated variation in axial field intensity is as in Fig. 10 .

Fig. 12. Radial variation $B_{T}$ of the vacuum magnetic field intensities in $\mathrm{TMX}$ for the conditions of Figs. 10 and 11 . The appropriate radial locations of the transmitting microwave horns for the EO-mode of propagation are also shown $[(a),(b)$, and (c) in Fig. 10$] . \theta$ refers to th: relative angle from the plane of the B-rfan.

Fig. 13. Expanded plot of $B_{T}$ vs radius $R$ at two fixed axial locations in TMX, $z$ $150 \mathrm{~cm}$ and $z=320 \mathrm{~cm} \quad[(a)$ and $(c)$, respectively, in Fig. 10 ]. 


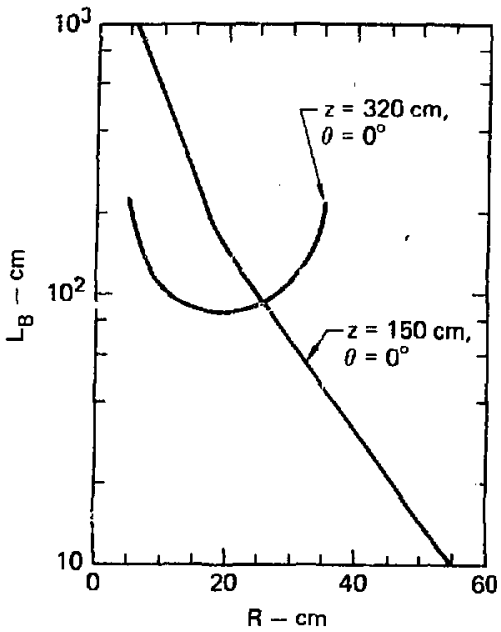

Fig. 14. Radial gradient scale length $L_{B}=B|\partial B / \partial X|^{-1}$ vo radius $R$ at two axial locations in TMX.

In Fig. 15, we show the calculated absorption at different radial positions at the center of the THX mirror plug at $z=320 \mathrm{~cm}$. Here we assumed that the magnetic field gradients are as given in Figs. 12 or 13 and that the density and temperature profiles obey the relationships

$$
\begin{aligned}
& n(r)=n_{0} \exp \left[-\left(R / 10^{2}\right)\right], \\
& T(r)=T(0) \exp \left|-\left(R / 10^{2}\right)\right| .
\end{aligned}
$$

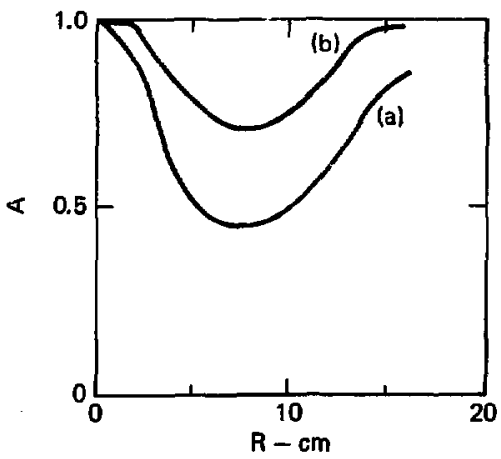

Fig. 15. Absorption coefricient A vs $R$ for ThX parameters for EO-mode, $\omega=\omega$ ce' where $f=28 \mathrm{GRz}$ at $R=2 \mathrm{~cm}, T_{c}(0)=$ $400 \mathrm{ev}, \mathrm{n}_{\mathrm{e}}(0)=1 \times 10^{13} \mathrm{~cm}^{-3}, \mathrm{z}=$ $320 \mathrm{~cm}, \mathrm{n}_{\mathrm{e}}(\mathrm{R})=\mathrm{n}_{0} \exp \left[-(\mathrm{k} / 10)^{2}\right]_{2}$ and $T(R)=T(0) \exp \left[-(R / 10)^{2}\right]$; $N_{z}=0.3$.

(a) one pass through one resonance layer; and

(b) one pass through two regonance layers. 
We further sook $N_{z}=0.3$ (so that the angle of incidence is $72^{\circ}$ ), and assumed that $T_{e}(0)=400 \mathrm{eV}, n_{e}(0)=1 \times 10^{13} \mathrm{~cm}^{-3}$, and $f_{0}=f_{c e} \operatorname{locally}$. In particular, $f_{0}=28 \mathrm{GHz}$ at $R \approx 2 \mathrm{~cm}$ at 1 ow values of $B$ in $T M X$.

Thus, we see that absorption efficiencies in excess of $80 \%$ are $0^{\circ}$, tained for $\mathrm{R}<4 \mathrm{~cm}$ when we consider one pass through the two resonance 1ayers. The localization of the absorption according to Eq. (15) is shown in Fig. 16 by the quantity $\Delta$. We see that when $R \cong 2.5 \mathrm{~cm}, \Delta \approx 2 R$; hence, we take this value of $R$ for estimating the maximum absorption efficiency, which in the present case is in excess of $90 \%$. We remark that accessibility exists in the whole region $z \approx 270$ to $350 \mathrm{~cm}$, and thus one night consider $z \approx 300 \mathrm{~cm}$ as a particularly suitable lacation for heating since here thr density is considerably lower than at $z=320 \mathrm{~cm}$.

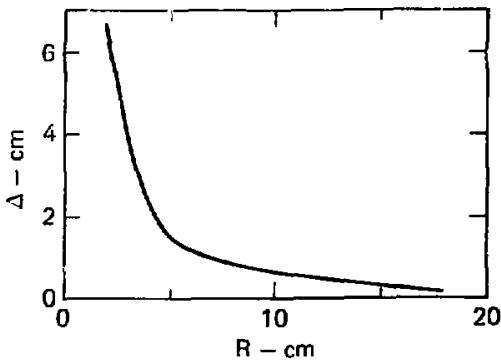

Fig. 16. Width of the absorption layer $\Delta$ vs radius $R$. Associated parameters are as 1 isted in Figs. 12 through 14.

Although these results were obtained for low $B$, at high $B$ the magnetic field would be depressed near the center and the corresponding plasma density may also be decreased. Taking

$$
B_{\text {actual }} \approx(l-B)^{1 / 2} \text { B }_{\text {vacuum }}
$$

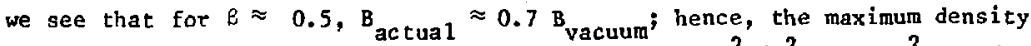
at accessibility would be reduced by the factor $1 \approx \omega_{p e}^{2} / \omega_{c e}^{2} \alpha n_{e} / B^{2} \alpha n_{e} / B B_{v}^{2}$ or by the value of $\beta$. Thus, $h i g h-\beta$ values will proportionally reduce the maximum density to which accessibility exists.

Heating the TMX central solenoid at high plasma densities at $z<150 \mathrm{~cm}$ would be particularly difficult at finite $\beta$. For example, in TMX the central vacuum solenoid may have a maximum field of $B=0.2 \mathrm{~T}$ (see Fig. 12). This wou id limit the frequency to $\mathrm{f}=\mathrm{f}_{\mathrm{ce}}=5.8 \mathrm{GHz}, \mathrm{f}=2 \mathrm{f}_{\mathrm{ce}}=11.6 \mathrm{GHz}$; thus, the accessible density would be $n \approx 3 \times 10^{11} \mathrm{~cm}^{-3}$ at $\mathrm{f}_{0}=\mathrm{f}_{\mathrm{ce}}$, and $\mathrm{n} \approx 10^{12} \mathrm{~cm}^{-3}$ 
at $E_{0}=2 f_{c e}$, both rather low values. Finite $B$ would further depress these' density values, and we thus conclude that electron cyclotron resonance heating (ECRH) in the center of the TMX solenoid is restricted to uninterestingly low values of the density. Therefore, heating the central cell at interesting densities (i.e., at $\mathrm{n}>5 \times 10^{12} \mathrm{~cm}^{-3}$ ) $\mathrm{could}$ only be performed in the rising portion of the magnetic field, ramely at $z \approx 210$ to $220 \mathrm{~cm}$ in Figs. 10 and 12 . This would require that the microwave horns be placed rather close to the axis, i.e., at $R<15 \mathrm{~cm}$. Because here typically $B_{\text {vacuum }}(R=0) \approx 1 \mathrm{~T}$, heating at $\mathrm{n}=10^{13} \mathrm{~cm}^{-3}$ and $\mathrm{F}=\mathrm{f}_{\mathrm{ce}}=28 \mathrm{GHz}$ appears feasible. We note that at $\mathrm{z}=$ $220 \mathrm{~cm}$ there are strong magnetic field gradients in the $z$-direction (larger than in the $x$-direction); inence, care must be exercised in injecting the microwave beam at the correct angle so as to absorb the microwave energy in the interior of the plasma (rather than at the plasma edge). We believe that detailed ray tracing would be of help to determine the correct angle of the microwave jorns when one considers heating plasma.

In Fig. 17 we show the mod-B curves in the solenoid-plug transition region at $z \approx 212$ to $222 \mathrm{~cm}$ and $\theta=90^{\circ}$. The half-width of the plasma in this region is typically $4 \mathrm{~cm}$. Thus, we see that good accessibility exists in this whole region for the EO-mode. . Since the radial magnetic field gradients in Fig. 17 are comparable to that in Fig. 14 (i.e., the center of the

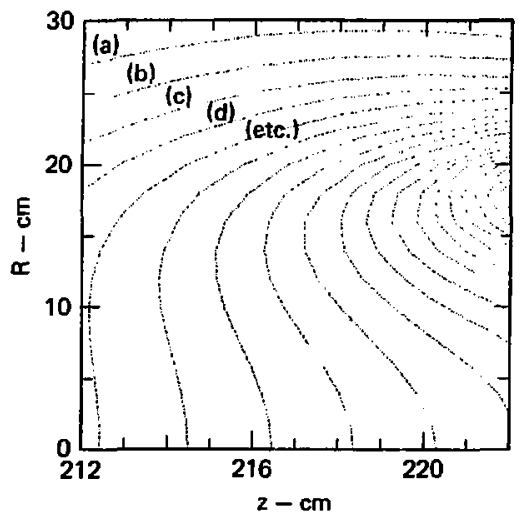

Fig. 17. Mod-B surfaces at $\theta=90^{\circ}, \triangle B=$ $0.072 \mathrm{~T}$, for the following values of $B$ :
(a) $0.6257 \mathrm{~T}$
(h) $0.1128 \mathrm{~T}$
(b) $0.6975 \mathrm{~T}$
(i) $0.1199 \mathrm{~T}$
(c) $0.7692 \mathrm{~T}$
(j) $0.1271 \mathrm{~T}$
(d) $0.8409 \mathrm{~T}$
(k) $0.1343 \mathrm{~T}$
(e) $0.9126 \mathrm{~T}$
(1) $0.1415 \mathrm{~T}$
(f) $0.9843 \mathrm{~T}$
(m) $0.1486 \mathrm{~T}$
(g) $0.1056 \mathrm{~T}$
(a) $0.1557 \mathrm{~T}$
(p) $0.1629 \mathrm{~T}$

$z-\mathrm{cm}$ 
plug), we expect comparable absorption at the same frequency and temperature. We also note that even the n-mode could be used to heat the plasma, although not with as great efficiency as the EO-mode. In future devices wtere $\mathrm{T}_{\mathrm{e}} \tilde{\nu} 1$ kev, the 0-mode will likely be also completely absorbed.

In Fig. 18, we show a general plot of frequencies and critical accessible densities as a function of magnetic field. For a given magnetic field one can directly determine the necessary frequency at either $f_{0}=f_{c e}$ or $f_{0}=$ $2 \mathrm{f}_{c e}$, and the associated critical (maximum accessible) density. This magnetic field should be taken as the net magnetic field, corrected for $B$. Thus, we see that in order to allow operations at higher densities, it would be advantagerus to work at the harmonic of the cyclotron freq ency. In IMX, for example, by working at $\mathrm{B}=10 \mathrm{~T}, \mathrm{f}_{0}=2 \mathrm{f}_{\mathrm{ce}}=56 \mathrm{GHz}$, a density of $\mathrm{n} \approx 2 \times 10^{13}$ is accessible $\therefore$ the plug; at $B=0.65 \mathrm{~T}, \mathrm{~F}_{0}=2 \mathrm{E}_{\mathrm{ce}}=36 \mathrm{GHz}$, a density of $\mathrm{n} \approx$ $1 \times 10^{13}$ is accessible toward the solenoid. In a device such as TMR, by working at $B=2.25 \mathrm{~T}, \mathrm{f}_{0}=2 \mathrm{~F}_{\mathrm{ce}}=125 \mathrm{GHz}$, a density of $\mathrm{n} \approx 1 \times 10^{14} \mathrm{~cm}^{-3}$ is accessible, which would correspond to typical values in the central solenoid. It is believed that such power sources may become available in the next 2 to 3 years. Taking a vacuum field of $B \approx 3.2 \mathrm{~T}, B \approx 0.5$, we obtain a local field of $B \approx 2.2 \mathrm{~T}$, witich may be a typical value half way in radially, even if $B \rightarrow 1$ near the center. The absorption at high temperatures ( $\mathrm{T}_{\mathrm{e}}>\mathrm{l} \mathrm{keV}$ ) is excellent at the second harmonic, even if the gradient scale lengths shorten due to the presence of the magnetic well.

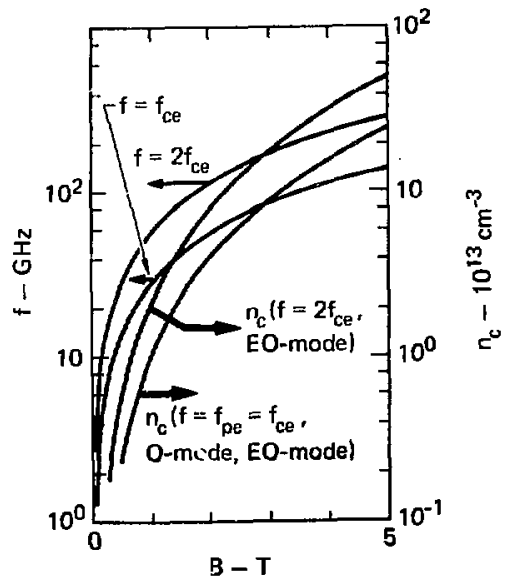

Fig. 18. Critical frequencies and cut-off densities $n_{c}$ vs magnetíc field. 
CONCLUS IONS

We have examined in some detail the predictions of the theory of electron cyclotron resonance heating of mirror devices. We found that heating with the extraordinary mode either near the fundamental or at the harmonir of the electron cyclotron frequency offered the best possibility for heating electrons in relatively low-density devices ( $\left.\omega_{p e}<\omega_{c e}\right)$. In present-day tandem devices, either the mirror plug or the rising portion of the mirror field between the center cell and the plug wffers the best place for heating electrons at $\omega_{0}=\omega_{c e} \approx \omega_{\text {pe }}$. Care must be exercised in selecting the proper angle of microwave injection.

In future devices where the magnetic field will be considerably higher, heating the center cell is feasible both at $w_{0}=w_{c e}$ and $w_{0}=2 w_{c e}$. Once $\mathrm{high-frequency} \mathrm{sources} \mathrm{become} \mathrm{available,} \mathrm{heating} \mathrm{at} \mathrm{the} \mathrm{harmonic} \mathrm{offers}$ the best possibility for heating the higher-density plasmas. In particular, at a central magnetic field of $\mathrm{B}>3.0 \mathrm{~T}, f>100 \mathrm{GHz}$, densities approaching $\mathrm{n} \approx 10^{14} \mathrm{~cm}^{-3}$ may be heated in devices with $\beta \approx 0.5$. In mosi cases considered, the absorption efficiencies were found to be very high $(n \approx 0.80)$. The heating efficiency will further increase at higher electron temperatures.

Finally, heating with whistler waves offers intaresting possibilities even in high-density devices $\left(\omega_{p e} \gg \omega_{c e}\right)$. However, the waves must travel through the mirror to the central cell, and nonlinear effects may play an important role in determining the absorption mechanisms and the resulting diatribution functions at the high-power levels considered here. Only experimental tests could determine how serious such effects may be.

\section{ACKNOWLEDGMENTS}

The autino: wishes to thank the many physicists at Lawrence Livermore Laboratory whe partcipated in enlightening discussions conceming tandem mirrors and the need for electron heating in such devices. Tarticular thanks are due to D. E. Baldwin, T. K. Fowler, B. G. Logan, T. G. Simonen, and W. C. Turner for the more detailed discussions. Special thanks are due to J. H. Fuoce, who provided the magnetic field plots of TMx. 


\section{REFERENCES}

1. M. S. Ioffe, B. I. Kanaev, V. P. Pastukhov and E. E. Yushmanov, Zh. Eksp. Teor. Fiz., 67, 2145 (1974); also in Proc. European Conf. Plasma Physics and Fusion Research (Prague, 1977), p. 99.

2. B. G. Logan, W. L. Barr, D. J. Bender, G. A. Carlson, W. L. S.xter, J. N. Doggett, R. S. Devoto, J. F. Fink, T. K. Fowler, G. W. Hamilton, B. Lappan, J. N. Lee, W. D. Neef, Jr., A. A. Mirin, R. W. Moir, M. A. Peterson, and M. E. Rensink, Tandem Mirror Reactors, Lawrence Livermore Laboratory, Rept. UCRL-80644 (1978); presented at the Fourth Intern. Conf. Driven Magnetic Fusion Reactors (Erice, Italy, 1978).

3. T. H. Stix, Nuci. Fusion 15, 737 (1975).

4. Calculations conceming electron heating by Alfuén waves in mirror reactors will be ciiscussed in a subsequent report.

5. Varian is developila a gyrotron with $f=28 \mathrm{GHz}, 200-\mathrm{kW}$ CW power; the Naval Research Laboratory is developing a 200-kW, 35-GHz, 10-ms pulse gyrotron.

6. N. I. Zaytsev, T. B. Pankratova, M. I. Petelin, and V. A. Flyagin, Radio Eng. Electron. Phys. 19, 103 (1974).

7. J. L. Hirshfie d and v. L. Granastien, IEEE Trans. Microwave Theory Tech. MIT 25, 513 (1977).

8. H. R. Jory, Varian Associates, private communication (1978).

9. V. V. Alikaev, et al., Sov. J. Plasma Phys. 2, 212 (1976).

10. 0. Eldridge, Phys. Fluids 15, 676 (1972).

11. J. C. Sprott, Fhys. Fluids 15, 2247 (1972).

12 A. J. Lichtenberg, and M. J. Schwartz, Plasma Phys. 11, 101 (1969).

13. R. A. Dand1, A. C. England, W. B. Ard, H. O. Eason, M. C. Becker, and G. M. Hass, Nuc1. Fusion 4, 344 (1964); H. Ikegami, H. Ikazi, M. Hosokawa, K. Takayama, and S. Tanaka, Phys. Fluidis 11, 1061 (1968); and R. A. Dandle, H. O. Eason, G. E. Guest, C. L. Hedrick, H. Ikegami, and D. B. ïsl scn, "Plasma Confinement and Heating in the Elmo Bumpy Torus (EBT)," ir. Proc. Fifth In i. Conf. on Plasma Phys. and Cont. Nucl. Fus. Res.. Tokyo, 1974 (IAEA, Vienna), Vol. 2, 141 (1974).

14. R. A. Blanken, T. H. Stix, and A. H'. Kuckes, Plasma Phys. 11, 945 (1969).

15. M. Porkolab, M. H. Brennan, and J. Sinnis, Plasma Phys. 13, 353 (1971). 
16. A. G. Lituak, G. V. Pernitin, E. V. Suvorov, and A. A. Frajman, Nucl. Fusion 17, 659 (1977).

17. I. Fidone, G. Granata, G. Ramponi, and R. L. Meyer, Phys. Fluids 21, 645 (1978).

18. 0. Eldridge, W. Namkung, and A. C. England, Electron Cyclotron Heating in Tokamaks, Oak Ridge National Laboratory, Rept. ORNL/RM-6052 (1977).

19. T. M. Antonsen, Jr., and W. M. Manheimer, Phys. Fluids 21, 2295 (1978).

20. T. H. Stix, The Theory of Plasma Waves (McGraw Hi11, New York, 1962), ch. 2 .

21. B. D. McVey and J. E. Scharer, Phys, Fluids 17, 142 (1974).

22. M. Portiolab, V. Arunasalam, and R. A. E11is, Jr., Phys, Rev. Lett. 29, 1438 (1972).

23. R. L. Stenzel and W. Gekelman, Phys. Fluids 20, 108 (1977).

24. 3. Foote, Lawrence Livermore Laboratory, private communication (July (978). 